\title{
Noncommutative Riemann Surfaces
}

\section{J.M. Isidro*}

Dip. di Fisica "G._Galilei" - INFN, Università di Padova, Via Marzolo 8, 35131 Padova, Italy. isidro@d.infn.it

ABSTRACT: We compactify $\mathrm{M}$ (atrix) theory on Riemann surfaces $\Sigma$ with genus $g>1$. Following [1], we construct a projective unitary representation of $\pi_{1}(\Sigma)$ realized on $L^{2}(\mathbf{H})$, with $\mathbf{H}$ the upper half-plane. As a first step we introduce a suitably gauged $\operatorname{sl}_{2}(\mathbf{R})$ algebra. Then a uniquely determined gauge connection provides the central extension which is a 2-cocycle of the 2nd Hochschild cohomology group. Our construction is the double-scaling limit $N \rightarrow \infty, k \rightarrow-\infty$ of the representation considered in the Narasimhan-Seshadri theorem, which represents the higher-genus analog of 't Hooft's clock and shift matrices of QCD. The concept of a noncommutative Riemann surface $\Sigma_{\theta}$ is introduced as a certain $C^{\star}$-algebra. Finally we investigate the Morita equivalence.

\section{Introduction}

The $P_{-}=N / R$ sector of the discrete light-cone quantization of uncompactified M-theory is given by the supersymmetric quantum mechanics of $U(N)$ matrices. The compactification of $\mathrm{M}($ atrix $)$

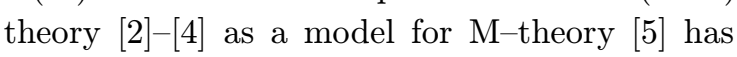

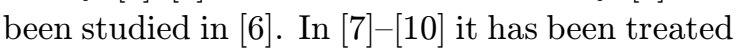
using noncommutative geometry [1] 1 in. These investigations apply to the $d$-dimensional torus $T^{d}$, and have been further dealt with from various

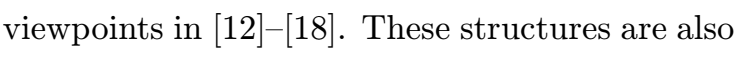
relevant in noncommutative string and gauge the-

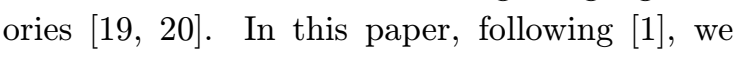
address the compactification M(atrix) theory on Riemann surfaces with genus $g>1$.

A Riemann surface $\Sigma$ of genus $g>1$ is constructed as the quotient $\mathbf{H} / \Gamma$, where $\mathbf{H}$ is the upper half-plane, and $\Gamma \subset \mathrm{PSL}_{2}(\mathbf{R}), \Gamma \cong \pi_{1}(\Sigma)$, is a Fuchsian group acting on $\mathbf{H}$ as

$$
\gamma=\left(\begin{array}{ll}
a & b \\
c & d
\end{array}\right) \in \Gamma, \quad \gamma z=\frac{a z+b}{c z+d} .
$$

In the absence of elliptic and parabolic generators, the $2 g$ Fuchsian generators $\gamma_{j}$ satisfy

$$
\prod_{j=1}^{g}\left(\gamma_{2 j-1} \gamma_{2 j} \gamma_{2 j-1}^{-1} \gamma_{2 j}^{-1}\right)=\mathbf{1} .
$$

*In collaboration with G. Bertoldi (MIT, USA), M. Matone and P. Pasti (Padova, Italy).
Inspired by $\mathrm{M}$ (atrix) theory, let us promote the complex coordinate $z=x+i y$ to an $N \times N$ complex matrix $Z=X+i Y$, with $X=X^{\dagger}$ and $Y=Y^{\dagger}$. This suggests defining fractional linear transformations of $Z$ through conjugation with some non-singular matrix $\mathcal{U}$ :

$$
\mathcal{U} Z \mathcal{U}^{-1}=(a Z+b \mathbf{1})(c Z+d \mathbf{1})^{-1} .
$$

Accordingly, operators $\mathcal{U}_{k}$ representing the Fuchsian generators $\gamma_{k}$ can be constructed, such that

$$
\prod_{k=1}^{g}\left(\mathcal{U}_{2 k-1} \mathcal{U}_{2 k} \mathcal{U}_{2 k-1}^{-1} \mathcal{U}_{2 k}^{-1}\right)=e^{2 \pi i \theta} \mathbf{1}
$$

While we will find the solution to (1.4), we will consider slightly different versions of (1.3il). This construction cannot be implemented for finite $N$, as taking the trace of (11.3i) shows. It can be interpreted as defining a sort of M(atrix) uniformization, in which the Möbius transformation of the $\mathrm{M}($ atrix) coordinate $Z$ is defined through (11.3i).

\section{Compactification in $g>1$}

Next we present an explicit Ansatz to compactify 11-dimensional supergravity on a Riemann surface with $g>1$. The Einstein equations read

$$
R_{M N}-\frac{1}{2} G_{M N} R
$$




$$
\begin{aligned}
=\frac{1}{3}( & H_{M L_{1} L_{2} L_{3}} H_{N L_{1}^{\prime} L_{2}^{\prime} L_{3}^{\prime}} G^{L_{1} L_{1}^{\prime}} G^{L_{2} L_{2}^{\prime}} G^{L_{3} L_{3}^{\prime}} \\
- & \frac{1}{8} G_{M N} H_{L_{1} L_{2} L_{3} L_{4}} H_{L_{1}^{\prime} L_{2}^{\prime} L_{3}^{\prime} L_{4}^{\prime}} \\
& \left.\times G^{L_{1} L_{1}^{\prime}} G^{L_{2} L_{2}^{\prime}} G^{L_{3} L_{3}^{\prime}} G^{L_{4} L_{4}^{\prime}}\right),
\end{aligned}
$$

where $H_{M N P Q}$ is the field strength of $C_{M N P}$. We try an Ansatz by diagonally decomposing $G_{M N}$ into 2-, 4- and 5-dimensional blocks, with $H_{M N P Q}$ taken along the 4-dimensional subspace:

$$
\begin{gathered}
G_{M N}=\operatorname{diag}\left(g_{\alpha \beta}^{(2)}, g_{m n}^{(4)}, g_{a b}^{(5)}\right), \\
H_{M P Q R}=\epsilon_{m p q r} f .
\end{gathered}
$$

The Einstein equations then decompose as

$$
\begin{aligned}
R_{i_{k} j_{k}}^{(k)}- & \frac{1}{2} g_{i_{k} j_{k}}^{(k)}\left(R^{(2)}+R^{(4)}+R^{(5)}\right) \\
= & \epsilon_{k} \operatorname{det} g^{(4)} f^{2} g_{i_{k} j_{k}}^{(k)},
\end{aligned}
$$

where $k=2,4,5,\left(i_{2}, j_{2}\right)=(\alpha, \beta),\left(i_{4}, j_{4}\right)=$ $(m, n),\left(i_{5}, j_{5}\right)=(a, b)$, and $\epsilon_{2}=\epsilon_{4}=-\epsilon_{5}=1$. Some manipulations lead to

$$
R^{(k)}=c_{k} f^{2} \operatorname{det} g^{(4)},
$$

with $c_{2}=-4 / 3, c_{4}=16 / 3$ and $c_{5}=-10 / 3$. We observe that $f=0$ would reproduce the toroidal case. A non-vanishing $f$ is a deformation producing $g>1$. It suffices that $g^{(4)}$ have positive signature for $R^{(2)}$ to be negative, as required in $g>1$. Then a choice for the $4-$ and 5-dimensional manifolds is $S^{4}$ and $A d S^{5}$.

\section{Differential representation of $\Gamma$}

\subsection{The unitary gauged operators}

For $n=-1,0,1$ and $e_{n}(z)=z^{n+1}$ we consider the $\operatorname{sl}_{2}(\mathbf{R})$ operators $\ell_{n}=e_{n}(z) \partial_{z}$. We define

$$
L_{n}=e_{n}^{-1 / 2} \ell_{n} e_{n}^{1 / 2}=e_{n}\left(\partial_{z}+\frac{1}{2} \frac{e_{n}^{\prime}}{e_{n}}\right)
$$

These satisfy

$$
\begin{gathered}
{\left[L_{m}, L_{n}\right]=(n-m) L_{m+n}, \quad\left[\bar{L}_{m}, L_{n}\right]=0} \\
{\left[L_{n}, f\right]=z^{n+1} \partial_{z} f}
\end{gathered}
$$

For $k=1,2, \ldots, 2 g$, consider the operators

$$
T_{k}=e^{\lambda_{-1}^{(k)}\left(L_{-1}+\bar{L}_{-1}\right)} e^{\lambda_{0}^{(k)}\left(L_{0}+\bar{L}_{0}\right)} e^{\lambda_{1}^{(k)}\left(L_{1}+\bar{L}_{1}\right)},
$$

with the $\lambda_{n}^{(k)}$ picked such that $T_{k} z T_{k}^{-1}=\gamma_{k} z=$ $\left(a_{k} z+b_{k}\right) /\left(c_{k} z+d_{k}\right)$ so that by (1.2.

$$
\prod_{k=1}^{g}\left(T_{2 k-1} T_{2 k} T_{2 k-1}^{-1} T_{2 k}^{-1}\right)=\mathbf{1} .
$$

On $L^{2}(\mathbf{H})$ we have the scalar product

$$
\langle\phi \mid \psi\rangle=\int_{\mathbf{H}} d \nu \bar{\phi} \psi
$$

where $d \nu(z)=i d z \wedge d \bar{z} / 2=d x \wedge d y$. The $T_{k}$ provide a unitary representation of $\Gamma$.

Next consider the gauged $\operatorname{sl}_{2}(\mathbf{R})$ operators

$$
\begin{array}{r}
\mathcal{L}_{n}^{(F)}=F(z, \bar{z}) L_{n} F^{-1}(z, \bar{z}) \\
=e_{n}\left(\partial_{z}+\frac{1}{2} \frac{e_{n}^{\prime}}{e_{n}}-\partial_{z} \ln F(z, \bar{z})\right),
\end{array}
$$

where $F(z, \bar{z})$ is an undetermined phase function, to be determined later on. The $\mathcal{L}_{n}^{(F)}$ also satisfy the algebra (3.2). The adjoint of $\mathcal{L}_{n}^{(F)}$ is given by

$$
\mathcal{L}_{n}^{(F) \dagger}=-F \overline{e_{n}^{1 / 2}} \partial_{\bar{z}} \overline{e_{n}^{1 / 2}} F^{-1},
$$

with $\mathcal{L}_{n}^{(F) \dagger}=-\overline{\mathcal{L}}_{n}^{\left(F^{-1}\right)}$. Finally we define

$$
\Lambda_{n}^{(F)}=\mathcal{L}_{n}^{(F)}-\mathcal{L}_{n}^{(F) \dagger}=\mathcal{L}_{n}^{(F)}+\overline{\mathcal{L}}_{n}^{\left(F^{-1}\right)} .
$$

The $\Lambda_{n}^{(F)}$ enjoy the fundamental property that both their chiral components are gauged in the same way by the function $F$, that is

$$
\Lambda_{n}^{(F)}=F\left(L_{n}+\bar{L}_{n}\right) F^{-1},
$$

while also satisfying the $\operatorname{sl}_{2}(\mathbf{R})$ algebra:

$$
\begin{array}{r}
{\left[\Lambda_{m}^{(F)}, \Lambda_{n}^{(F)}\right]=(n-m) \Lambda_{m+n}^{(F)},} \\
{\left[\Lambda_{n}^{(F)}, f\right]=\left(z^{n+1} \partial_{z}+\bar{z}^{n+1} \partial_{\bar{z}}\right) f .}
\end{array}
$$

It holds that

$$
e^{\Lambda_{n}^{(F)}}=F e^{L_{n}+\bar{L}_{n}} F^{-1},
$$

which is a unitary operator since $\Lambda_{n}^{(F) \dagger}=-\Lambda_{n}^{(F)}$.

Let $b$ be a real number, and $A$ a Hermitean connection 1 -form to be identified presently. Set

$$
\mathcal{U}_{k}=e^{i b \int_{z}^{\gamma_{k} z} A} T_{k}
$$

where the integration contour is taken to be the Poincaré geodesic connecting $z$ and $\gamma_{k} z$. As the gauging functions introduced in (3.6i) we will take the functions $F_{k}(z, \bar{z})$ that solve the equation

$$
F_{k} T_{k} F_{k}^{-1}=e^{i b \int_{z}^{\gamma_{k} z} A} T_{k},
$$

that is

$$
F_{k}\left(\gamma_{k} z, \gamma_{k} \bar{z}\right)=e^{-i b \int_{z}^{\gamma_{k} z} A} F_{k}(z, \bar{z}) .
$$




\subsection{The gauged algebra}

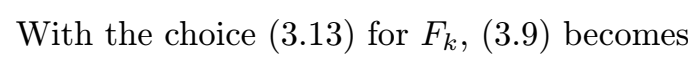

$$
\begin{aligned}
& \Lambda_{n, k}^{(F)}=F_{k}\left(L_{n}+\bar{L}_{n}\right) F_{k}^{-1} \\
= & z^{n+1}\left(\partial_{z}+\frac{n+1}{2 z}-\partial_{z} \ln F_{k}\right) \\
+ & \bar{z}^{n+1}\left(\partial_{\bar{z}}+\frac{n+1}{2 \bar{z}}-\partial_{\bar{z}} \ln F_{k}\right) .
\end{aligned}
$$

The $\Lambda_{n, k}^{(F)}$ satisfy the algebra

$$
\begin{gathered}
{\left[\Lambda_{m, j}^{(F)}, \Lambda_{n, k}^{(F)}\right]} \\
=(n-m) \Lambda_{m+n, j}^{(F)}+F_{k}^{-1}\left|e_{n}\right| \Lambda_{n, k}^{(F)}\left|e_{n}\right|^{-1} F_{k} \\
\times F_{j}^{-1}\left|e_{m}\right| \Lambda_{m, j}^{(F)}\left|e_{m}\right|^{-1} F_{j}\left(\ln F_{j}-\ln F_{k}\right), \\
{\left[\Lambda_{n, k}^{(F)}, f\right]=\left(z^{n+1} \partial_{z}+\bar{z}^{n+1} \partial_{\bar{z}}\right) f .}
\end{gathered}
$$

Upon exponentiating $\Lambda_{n, k}^{(F)}$ one finds

$$
\mathcal{U}_{k}=e^{\lambda_{-1}^{(k)} \Lambda_{-1, k}^{(F)}} e^{\lambda_{0}^{(k)} \Lambda_{0, k}^{(F)}} e^{\lambda_{1}^{(k)} \Lambda_{1, k}^{(F)}}
$$

that is, the $\mathcal{U}_{k}$ are unitary, and

$$
\mathcal{U}_{k}^{-1}=T_{k}^{-1} e^{-i b \int_{z}^{\gamma_{k} z} A}=e^{-i b \int_{\gamma_{k}^{-1} z}^{z} A} T_{k}^{-1} .
$$

\subsection{Computing the phase}

It is immediate to see that the $\mathcal{U}_{k}$ defined in $(\overline{3} . \overline{1} \overline{2})$ satisfy $(\overline{1} . \overline{4})$ for a certain value of $\theta$ :

$$
\begin{aligned}
& \prod_{k=1}^{g}\left(\mathcal{U}_{2 k-1} \mathcal{U}_{2 k} \mathcal{U}_{2 k-1}^{\dagger} \mathcal{U}_{2 k}^{\dagger}\right)=e^{i b \int_{z}^{\gamma_{1} z} A_{1}} T_{1} \\
& \times e^{i b \int_{z}^{\gamma_{2} z} A} T_{2} e^{-i b \int_{\gamma_{1}^{-1} z}^{z} A} T_{1}^{-1} e^{-i b \int_{\gamma_{2}^{-1} z}^{z} A} T_{2}^{-1} \ldots \\
& =\exp \left[i b\left(\int_{z}^{\gamma_{1} z} A+\int_{\gamma_{1} z}^{\gamma_{2} \gamma_{1} z} A\right)\right] \times \\
& \exp \left[i b\left(\int_{\gamma_{2} \gamma_{1} z}^{\gamma_{1}^{-1} \gamma_{2} \gamma_{1} z} A+\int_{\gamma_{1}^{-1} \gamma_{2} \gamma_{1} z}^{\gamma_{2}^{-1} \gamma_{1}^{-1} \gamma_{2} \gamma_{1} z} A+\ldots\right)\right] \\
& \times \prod_{k=1}^{g}\left(T_{2 k-1} T_{2 k} T_{2 k-1}^{-1} T_{2 k}^{-1}\right)=e^{i b \oint_{\partial \mathcal{F}_{z}} A},
\end{aligned}
$$

where $\mathcal{F}_{z}=\left\{z, \gamma_{1} z, \gamma_{2} \gamma_{1} z, \gamma_{1}^{-1} \gamma_{2} \gamma_{1} z, \ldots\right\}$ is a fundamental domain for $\Gamma$. The basepoint $z$, plus the action of the Fuchsian generators on it, determine $\mathcal{F}_{z}$, as the vertices are joined by geodesics.

\subsection{Uniqueness of the gauge connection}

For (13. $\overline{1} \overline{9})$ to provide a projective unitary representation of $\Gamma, \int_{\mathcal{F}_{z}} d A$ should be $z$-independent. Changing $z$ to $z^{\prime}$ can be expressed as $z \rightarrow z^{\prime}=\mu z$ for some $\mu \in \mathrm{PSL}_{2}(\mathbf{R})$. Then $\mathcal{F}_{z} \rightarrow \mathcal{F}_{\mu z}=$ $\left\{\mu z, \gamma_{1} \mu z, \gamma_{2} \gamma_{1} \mu z, \gamma_{1}^{-1} \gamma_{2} \gamma_{1} \mu z, \ldots\right\}$. Now consider $\mathcal{F}_{z} \rightarrow \mu \mathcal{F}_{z}=\left\{\mu z, \mu \gamma_{1} z, \mu \gamma_{2} \gamma_{1} z, \mu \gamma_{1}^{-1} \gamma_{2} \gamma_{1} z, \ldots\right\}$ The congruence $\mu \mathcal{F}_{z} \cong \mathcal{F}_{\mu z}$ follows from two facts: that the vertices are joined by geodesics, and that $\mathrm{PSL}_{2}(\mathbf{R})$ maps geodesics into geodesics. Since $\Gamma$ is defined up to conjugation, $\Gamma \rightarrow \mu \Gamma \mu^{-1}$, if $\mu \mathcal{F}_{z}$ is a fundamental domain, so is $\mathcal{F}_{\mu z}$. Thus, to have $z$-independence we need $\forall \mu \in \mathrm{PSL}_{2}(\mathbf{R})$

$$
\int_{\mathcal{F}_{z}} d A=\int_{\mathcal{F}_{\mu z}} d A=\int_{\mu \mathcal{F}_{z}} d A=\int_{\mathcal{F}} d A .
$$

This fixes the $(1,1)$-form $d A$ to be $\mathrm{PSL}_{2}(\mathbf{R})$ invariant. It is well known that the Poincaré form is the unique $\mathrm{PSL}_{2}(\mathbf{R})$-invariant $(1,1)$-form, up to an overall constant factor. This is a particular case of a more general fact [2]1]. The Poincaré metric $d s^{2}=y^{-2}|d z|^{2}=2 g_{z \bar{z}}|d z|^{2}=e^{\varphi}|d z|^{2}$ has curvature $R=-g^{z \bar{z}} \partial_{z} \partial_{\bar{z}} \ln g_{z \bar{z}}=-1$, so that $\int_{\mathcal{F}} d \nu e^{\varphi}=-2 \pi \chi(\Sigma)$, where $\chi(\Sigma)=2-2 g$ is the Euler characteristic. As the Poincaré $(1,1)$-form is $d A=e^{\varphi} d \nu$, this uniquely determines the gauge field to be

$$
A=A_{z} d z+A_{\bar{z}} d \bar{z}=d x / y
$$

up to gauge transformations. Using $\oint_{\partial \mathcal{F}} A=$

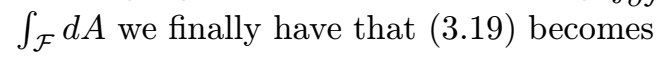

$$
\prod_{k=1}^{g}\left(\mathcal{U}_{2 k-1} \mathcal{U}_{2 k} \mathcal{U}_{2 k-1}^{\dagger} \mathcal{U}_{2 k}^{\dagger}\right)=e^{2 \pi i b \chi(\Sigma)} .
$$

\subsection{Non-Abelian extension}

Up to now we considered the case in which the connection is Abelian. However, it is easy to extend our construction to the non-Abelian case in which the gauge group $U(1)$ is replaced by $U(N)$. The operators $\mathcal{U}_{k}$ now become

$$
\mathcal{U}_{k}=P e^{i b \int_{z}^{\gamma_{k} z} A_{1}} T_{k},
$$

where the $T_{k}$ are the same as before, times the $N \times N$ identity matrix. Eq. $\left(\underline{3}_{-}^{-1} \cdot \overline{9^{\prime}}\right)$ is replaced by

$$
\prod_{k=1}^{g}\left(\mathcal{U}_{2 k-1} \mathcal{U}_{2 k} \mathcal{U}_{2 k-1}^{\dagger} \mathcal{U}_{2 k}^{\dagger}\right)=P e^{i b \oint_{\partial \mathcal{F}_{z}}{ }^{A}}
$$


Given an integral along a closed contour $\sigma_{z}$ with basepoint $z$, the path-ordered exponentials for a connection $A$ and its gauge transform $A^{U}=$ $U^{-1} A U+U^{-1} d U$ are related by [22

$$
\begin{gathered}
P e^{i \oint_{\sigma z} A}=U(z) P e^{i \oint_{\sigma_{z}} A^{U}} U^{-1}(z)= \\
U(z) P e^{i \oint_{\sigma_{z}} d \sigma^{\mu} \int_{0}^{1} d s s \sigma^{\nu} U^{-1}(s \sigma) F_{\nu \mu}(s \sigma) U(s \sigma)} U^{-1}(z) .
\end{gathered}
$$

Applying this to (13.24), we see that the only possibility to get a coordinate-independent phase is for the curvature $(1,1)-$ form $F=d A+[A, A] / 2$ to be the identity matrix in the gauge indices times a $(1,1)$-form $\eta$, that is $F=\eta \mathbf{1}$. It follows that

$$
P e^{i b \oint_{\partial \mathcal{F}} A}=e^{i b \int_{\mathcal{F}} F} .
$$

However, the above is only a necessary condition for coordinate-independence. Nevertheless, we can apply the same reasoning as in the Abelian case to see that $\eta$ should be proportional to the Poincaré (1,1)-form. Denoting by $E$ the vector bundle on which $A$ is defined, we have $k=$ $\operatorname{deg}(E)=\frac{1}{2 \pi} \operatorname{tr} \int_{\mathcal{F}} F$. Set $\mu(E)=k / N$ so that $\int_{\mathcal{F}} F=2 \pi \mu(E) \mathbf{1}$ and $\eta=-\frac{\mu(E)}{\chi(\Sigma)} e^{\varphi} d \nu$, i.e.

$$
F=2 \pi \mu(E) \omega \mathbf{1},
$$

where $\omega=\left(e^{\varphi} / \int_{\mathcal{F}} d \nu e^{\varphi}\right) d \nu$. Thus, by $\left(\overline{\bar{\beta}} . \overline{2} \overline{6}_{1}^{4}\right)$ we have that Eq.(B. (3.) becomes

$$
\prod_{k=1}^{g}\left(\mathcal{U}_{2 k-1} \mathcal{U}_{2 k} \mathcal{U}_{2 k-1}^{\dagger} \mathcal{U}_{2 k}^{\dagger}\right)=e^{2 \pi i b \mu(E)} \mathbf{1}
$$

which provides a projective unitary representation of $\pi_{1}(\Sigma)$ on $L^{2}\left(\mathbf{H}, \mathbf{C}^{N}\right)$.

\subsection{The gauge length}

A basic object is the gauge length function

$$
d_{A}(z, w)=\int_{z}^{w} A
$$

where the contour integral is along the Poincaré geodesic connecting $z$ and $w$. In the Abelian case

$$
d_{A}(z, w)=\int_{\operatorname{Re} z}^{\operatorname{Re} w} \frac{d x}{y}=-i \ln \left(\frac{z-\bar{w}}{w-\bar{z}}\right)
$$

which is equal to the angle $\alpha_{z w}$ spanned by the arc of geodesic connecting $z$ and $w$. Observe that the gauge length of the geodesic connecting two punctures, i.e. two points on the real line, is $\pi$. This is to be compared with the usual divergence of the Poincaré distance. Under a $\mathrm{PSL}_{2}(\mathbf{R})-$ transformation $\mu$, we have $\left(\mu_{x} \equiv \partial_{x} \mu x\right)$

$$
d_{A}(\mu z, \mu w)=d_{A}(z, w)-\frac{i}{2} \ln \left(\frac{\mu_{z} \bar{\mu}_{w}}{\bar{\mu}_{z} \mu_{w}}\right) .
$$

Therefore, the gauge length of an $n$-gon

$$
d_{A}^{(n)}\left(\left\{z_{k}\right\}\right)=\sum_{k=1}^{n} d_{A}\left(z_{k}, z_{k+1}\right)=\pi(n-2)-\sum_{k=1}^{n} \alpha_{k},
$$

where $z_{n+1} \equiv z_{1}, n \geq 3$, and $\alpha_{k}$ are the internal angles, is $\mathrm{PSL}_{2}(\mathbf{R})$-invariant. One can check that the $\mathrm{PSL}_{2}(\mathbf{R})$-transformation $(\overline{3} . \overline{3} \overline{1})$ corresponds to a gauge transformation of $A$. Furthermore, as we will see, the triangle length, that by Stokes' theorem corresponds to the Poincaré area, is proportional to the Hochschild 2-cocycle.

\subsection{Pre-automorphic forms}

A related reason for the relevance of the gauge length function is that it also appears in the definition of the $F_{k}$. The latter, which apparently never appeared in the literature before, are of particular interest. Let us recast $(\overline{3} \cdot \overline{1} \overline{1})$ as

$$
F_{k}\left(\gamma_{k} z, \gamma_{k} \bar{z}\right)=\left(\frac{\gamma_{k} z-\bar{z}}{z-\gamma_{k} \bar{z}}\right)^{b} F_{k}(z, \bar{z}) .
$$

Since $\left(\gamma_{k} z-\bar{z}\right) /\left(z-\gamma_{k} \bar{z}\right)$ transforms as an automorphic form under $\Gamma$, we call the $F_{k}$ preautomorphic forms. Eq.(13.14) indicates that finding the most general solution to ( $13 . \overline{3} \overline{3})$ is a problem in geodesic analysis. In the case of the inversion $\gamma_{k} z=-1 / z$ and $b$ an even integer, a solution

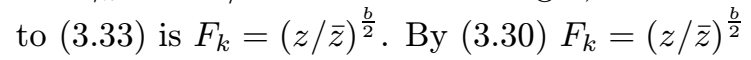
is related to the $A$-length of the geodesic connecting $z$ and 0 :

$$
e^{\frac{i}{2} b \int_{z}^{0} A}=F_{k}(z, \bar{z})=(z / \bar{z})^{\frac{b}{2}} .
$$

An interesting formal solution to $(\overline{3} . \overline{3} \overline{3})$ is

$$
F_{k}(z, \bar{z})=\prod_{j=0}^{\infty}\left(\frac{\gamma_{k}^{-j} z-\gamma_{k}^{-j-1} \bar{z}}{\gamma_{k}^{-j-1} z-\gamma_{k}^{-j} \bar{z}}\right)^{b} \text {. }
$$

To construct other solutions, we consider the uniformizing map $J_{\mathbf{H}}: \mathbf{H} \longrightarrow \Sigma$, which enjoys the property $J_{\mathbf{H}}(\gamma z)=J_{\mathbf{H}}(z), \forall \gamma \in \Gamma$. Then, if $F_{k}$ satisfies $\left(3.33^{i}\right)$, this equation is invariant under $F_{k} \rightarrow G\left(J_{\mathbf{H}}, \bar{J}_{\mathbf{H}}\right) F_{k}$. Since $\left|F_{k}\right|=1$, we should require $|G|=1$, otherwise $G$ is arbitrary. 


\section{Hochschild cohomology of $\Gamma$}

The Fuchsian generators $\gamma_{k} \in \Gamma$ are projectively represented by means of unitary operators $\mathcal{U}_{k}$ acting on $L^{2}(\mathbf{H})$. The product $\gamma_{k} \gamma_{j}$ is represented by ${ }^{1} \mathcal{U}_{j k}$, which equals $\mathcal{U}_{j} \mathcal{U}_{k}$ up to a phase:

$$
\mathcal{U}_{j} \mathcal{U}_{k}=e^{2 \pi i \theta(j, k)} \mathcal{U}_{j k}
$$

Associativity implies

$$
\theta(j, k)+\theta(j k, l)=\theta(j, k l)+\theta(k, l) .
$$

We can easily determine $\theta(j, k)$ :

$$
\begin{gathered}
\mathcal{U}_{j} \mathcal{U}_{k}=\exp \left(i b \int_{z}^{\gamma_{j} z} A+i b \int_{\gamma_{j} z}^{\gamma_{k} \gamma_{j} z} A\right) \times \\
\exp \left(-i b \int_{z}^{\gamma_{k} \gamma_{j} z} A\right) \mathcal{U}_{j k}=\exp \left(i b \int_{\tau_{j k}} A\right) \mathcal{U}_{j k},
\end{gathered}
$$

where $\tau_{j k}$ denotes the geodesic triangle with vertices $z, \gamma_{j} z$ and $\gamma_{k} \gamma_{j} z$. This identifies $\theta(j, k)$ as the gauge length of the perimeter of the geodesic triangle $\tau_{j k}$. By Stokes' theorem this is the Poincaré area of the triangle. A similar phase, introduced independently of any gauge connection, has been considered in [23- in the context of Berezin's quantization of $\mathbf{H}$ and Von Neumann algebras.

The information on the compactification of $\mathrm{M}$ (atrix) theory is encoded in the action of $\Gamma$ on $\mathbf{H}$, plus a projective representation of $\Gamma$. The latter amounts to the choice of a phase. Physically inequivalent choices of $\theta(j, k)$ turn out to be in one-to-one correspondence with elements in the 2nd Hochschild cohomology group $H^{2}(\Gamma, U(1))$ of $\Gamma$. This cohomology group is defined as follows. A $k$-cochain is an angular-valued function $f\left(\gamma_{1}, \ldots, \gamma_{k}\right)$ with $k$ arguments in $\Gamma$. The coboundary operator $\delta$ maps the $k$-cochain $f$ into the $(k+1)$-cochain $\delta f$ defined as

$$
\begin{gathered}
(\delta f)\left(\gamma_{0}, \ldots, \gamma_{k}\right)=f\left(\gamma_{1}, \ldots, \gamma_{k}\right) \\
+\sum_{l=1}^{k}(-1)^{l} f\left(\gamma_{0}, \ldots, \gamma_{l-1} \gamma_{l}, \ldots, \gamma_{k}\right) \\
\quad+(-1)^{k+1} f\left(\gamma_{0}, \ldots, \gamma_{k-1}\right) .
\end{gathered}
$$

${ }^{1}$ The differential representation of $\mathrm{PSL}_{2}(\mathbf{R})$ acts in reverse order with respect to the one by matrices.
Clearly $\delta^{2}=0$. A $k$-cochain annihilated by $\delta$ is called a $k$-cocycle. $H^{k}(\Gamma, U(1))$ is the group of equivalence classes of $k$-cocycles modulo the coboundary of $(k-1)$-cochains. The associativity condition (4.2) is just $\delta \theta(j, k)=0$. Thus $\theta$ is a 2-cocycle of the Hochschild cohomology. Projective representations of $\Gamma$ are classified by $H^{2}(\Gamma, U(1))=U(1)$. Hence $\theta=b \chi(\Sigma)$ is the unique parameter for this compactification $(\theta=$ $b \mu(E)$ in the general case).

\section{Stable bundles and double scaling limit}

We now present some facts about projective, unitary representations of $\Gamma$ and the theory of holomorphic vector bundles [24, $25 \overline{1}$. Let $E \rightarrow \Sigma$ be a holomorphic vector bundle over $\Sigma$ of rank $N$ and degree $k$. The bundle $E$ is called stable if the inequality $\mu\left(E^{\prime}\right)<\mu(E)$ holds for every proper holomorphic subbundle $E^{\prime} \subset E$. We may take $-N<k \leq 0$. We will further assume that $\Gamma$ contains a unique primitive elliptic element $\gamma_{0}$ of order $N$ (i.e., $\gamma_{0}^{N}=\mathbf{1}$ ), with fixed point $z_{0} \in \mathbf{H}$ that projects to $x_{0} \in \Sigma$.

Given the branching order $N$ of $\gamma_{0}$, let $\rho$ : $\Gamma \rightarrow U(N)$ be an irreducible unitary representation. It is said admissible if $\rho\left(\gamma_{0}\right)=e^{-2 \pi i k / N} \mathbf{1}$. Putting the elliptic element on the right-hand

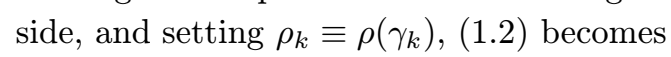

$$
\prod_{j=1}^{g}\left(\rho_{2 j-1} \rho_{2 j} \rho_{2 j-1}^{-1} \rho_{2 j}^{-1}\right)=e^{2 \pi i k / N} \mathbf{1}
$$

On the trivial bundle $\mathbf{H} \times \mathbf{C}^{N} \rightarrow \mathbf{H}$ there is an action of $\Gamma:(z, v) \rightarrow(\gamma z, \rho(\gamma) v)$. This defines the quotient bundle

$$
\mathbf{H} \times \mathbf{C}^{N} / \Gamma \rightarrow \mathbf{H} / \Gamma \cong \Sigma .
$$

Any admissible representation determines a holomorphic vector bundle $E_{\rho} \rightarrow \Sigma$ of rank $N$ and degree $k$. When $k=0, E_{\rho}$ is simply the quotient bundle (5.21) of $\mathbf{H} \times \mathbf{C}^{N} \rightarrow \mathbf{H}$. The NarasimhanSeshadri (NS) theorem [ $\left[2 \overline{6}_{1}\right]$ now states that a holomorphic vector bundle $E$ over $\Sigma$ of rank $N$ and degree $k$ is stable if and only if it is isomorphic to a bundle $E_{\rho}$, where $\rho$ is an admissible representation of $\Gamma$. Moreover, the bundles $E_{\rho_{1}}$ 
and $E_{\rho_{2}}$ are isomorphic if and only if the representations $\rho_{1}$ and $\rho_{2}$ are equivalent.

The standard Hermitean metric on $\mathbf{C}^{N}$ gives a metric on $\mathbf{H} \times \mathbf{C}^{N} \rightarrow \mathbf{H}$. This metric and the corresponding connection are invariant with respect to the action $(z, v) \rightarrow(\gamma z, \rho(\gamma) v)$, when $\rho$ is admissible. Hence they determine a (degenerate) metric $g_{N S}$ and a connection $A_{N S}$ on the bundle $E=E_{\rho}$. The connection $A_{N S}$ is compatible with the metric $g_{N S}$ and with the holomorphic structure on $E$, but it has a singularity at the branching point $x_{0} \in \Sigma$ of the covering $\mathbf{H} \rightarrow \Sigma$. The curvature $F_{N S}$ of $A_{N S}$ is a $(1,1)$-current with values in the bundle End $E$, characterized by the property ${ }^{2}$

$$
\int_{\Sigma} f \wedge F_{N S}=-2 \pi i \mu(E) \operatorname{tr} f\left(x_{0}\right),
$$

for every smooth section $f$ of the bundle End $E$. The connection $A_{N S}$ is uniquely determined by the curvature condition (5.3i) and by the fact that it corresponds to the degenerate metric $g_{N S}$. The connection $A_{N S}$ on the stable bundle $E=E_{\rho}$ is called the NS connection.

A differential-geometric approach to stability has been given by Donaldson [27] mitean metric on $\Sigma$, for example the Poincaré metric, normalized so that the area of $\Sigma$ equals 1. Let us denote by $\omega$ its associated $(1,1)$-form. A holomorphic bundle $E$ is stable if and only if there exists on $E$ a metric connection $A_{D}$ with central curvature $F_{D}=-2 \pi i \mu(E) \omega \mathbf{1}$; such a connection $A_{D}$ is unique.

The unitary projective representations of $\Gamma$ we constructed above have a uniquely defined gauge field whose curvature is proportional to the volume form on $\Sigma$. With respect to the representation considered by NS, we note that NS introduced an elliptic point to produce the phase, while in our case the latter arises from the gauge length. Our construction is directly connected with Donaldson's approach as $F=i F_{D}$, where $F$ is the curvature $(\overline{3} \cdot \overline{2} \overline{7})$. However, the main difference is that our operators are unitary differential operators on $L^{2}\left(\mathbf{H}, \mathbf{C}^{N}\right)$ instead of unitary matrices on $\mathbf{C}^{N}$. This allowed us to obtain a non-trivial phase also in the Abelian case.

\footnotetext{
${ }^{2}$ Note that our convention for $A$ differs from the one in the mathematical literature by a factor $i$.
}

It is however possible to understand the formal relation between our operators and those of NS. To see this we consider the adjoint representation of $\Gamma$ on End $\mathbf{C}^{N}$,

$$
\operatorname{Ad} \rho(\gamma) Z=\rho(\gamma) Z \rho^{-1}(\gamma)
$$

where $Z \in$ End $\mathbf{C}^{N}$ is understood as an $N \times N$ matrix. Let us also consider the trivial bundle $\mathbf{H} \times$ End $\mathbf{C}^{N} \rightarrow \mathbf{H}$. There is an action of $\Gamma$ : $(z, Z) \mapsto(\gamma z, \operatorname{Ad} \rho(\gamma) Z)$ that defines the quotient bundle

$$
\mathbf{H} \times \text { End } \mathbf{C}^{N} / \Gamma \rightarrow \mathbf{H} / \Gamma \cong \Sigma
$$

Then, the idea is to consider a vector bundle $E^{\prime}$ in the double scaling limit $N^{\prime} \rightarrow \infty, k^{\prime} \rightarrow-\infty$, with $\mu\left(E^{\prime}\right)=k^{\prime} / N^{\prime}$ fixed, that is

$$
\mu\left(E^{\prime}\right)=b \mu(E)
$$

In this limit, fixing a basis in $L^{2}\left(\mathbf{H}, \mathbf{C}^{N}\right)$, the matrix elements of our operators can be identified with those of $\rho(\gamma)$.

\section{Noncommutative Riemann surfaces}

Let us now introduce two copies of the upper half-plane, one with coordinates $z$ and $\bar{z}$, the other with coordinates $w$ and $\bar{w}$. While the coordinates $z$ and $\bar{z}$ are reserved to the operators $\mathcal{U}_{k}$ we introduced previously, we reserve $w$ and $\bar{w}$ to construct a new set of operators. We now introduce noncommutative coordinates expressed in terms of the covariant derivatives

$$
W=\partial_{w}+i A_{w}, \quad \bar{W}=\partial_{\bar{w}}+i A_{\bar{w}},
$$

with $A_{w}=A_{\bar{w}}=1 /(2 \operatorname{Im} w)$, so that

$$
[W, \bar{W}]=i F_{w \bar{w}},
$$

where $F_{w \bar{w}}=i /\left[2(\operatorname{Im} w)^{2}\right]$. Let us consider the following realization of the $\mathrm{sl}_{2}(\mathbf{R})$ algebra:

$$
\begin{gathered}
\hat{L}_{-1}=-w, \quad \hat{L}_{0}=-\frac{1}{2}\left(w \partial_{w}+\partial_{w} w\right), \\
\hat{L}_{1}=-\partial_{w} w \partial_{w} .
\end{gathered}
$$

We then define the unitary operators

$$
\hat{T}_{k}=e^{\lambda_{-1}^{(k)}\left(\hat{L}_{-1}+\overline{\hat{L}}_{-1}\right)} e^{\lambda_{0}^{(k)}\left(\hat{L}_{0}+\overline{\hat{L}}_{0}\right)} e^{\lambda_{1}^{(k)}\left(\hat{L}_{1}+\overline{\hat{L}}_{1}\right)},
$$


where the $\lambda_{n}^{(k)}$ are as in (3.3). Set $\mathcal{V}_{k}=\hat{T}_{k} \mathcal{U}_{k}$. Since the $\hat{T}_{k}$ satisfy (3.4), it follows that the $\mathcal{V}_{k}$ satisfy $(\underline{3} . \overline{2} \overline{2})$ and

$$
\mathcal{V}_{k} \partial_{w} \mathcal{V}_{k}^{-1}=\hat{T}_{k} \partial_{w} \hat{T}_{k}^{-1}=\frac{a_{k} \partial_{w}+b_{k}}{c_{k} \partial_{w}+d_{k}} .
$$

Setting $W=G \partial_{w} G^{-1}$, i.e. $G=(w-\bar{w})^{2}$, and using $A f(B) A^{-1}=f\left(A B A^{-1}\right)$, we see that

$$
\mathcal{V}_{k} W \mathcal{V}_{k}^{-1}=\hat{T}_{k} W \hat{T}_{k}^{-1}=G(\tilde{w}) \hat{T}_{k} \partial_{w} \hat{T}_{k}^{-1} G^{-1}(\tilde{w}),
$$

where

$$
\begin{gathered}
\tilde{w}=\hat{T}_{k} w \hat{T}_{k}^{-1}=-e^{-\lambda_{0}^{(k)}}+2 \lambda_{1}^{(k)}\left(\hat{L}_{0}-\lambda_{-1}^{(k)} w\right) \\
-\lambda_{1}^{(k) 2} e^{\lambda_{0}^{(k)}}\left(\hat{L}_{1}+2 \lambda_{-1}^{(k)} \hat{L}_{0}-\lambda_{-1}^{(k) 2} w\right)
\end{gathered}
$$

and by $(\overline{6} \cdot \overline{2} .5)$

$$
\mathcal{V}_{k} W \mathcal{V}_{k}^{-1}=\hat{T}_{k} W \hat{T}_{k}^{-1}=\frac{a_{k} \tilde{W}+b_{k}}{c_{k} \tilde{W}+d_{k}}
$$

where $\tilde{W}$ differs from $W$ by the connection

$$
\tilde{W}=\partial_{w}+G(\tilde{w})\left[\partial_{w} G^{-1}(\tilde{w})\right] .
$$

\subsection{Morita equivalence and large $N$ limit}

By a natural generalization of the $n$-dimensional noncommutative torus, one defines a noncommutative Riemann surface $\Sigma_{\theta}$ in $g>1$ to be an associative algebra with involution having unitary generators $\mathcal{U}_{k}$ obeying the relation (3.2. 2 . $)$. Such an algebra is a $C^{\star}$-algebra, as it admits a faithful unitary representation on $L^{2}\left(\mathbf{H}, \mathbf{C}^{N}\right)$ whose image is norm-closed. Relation (3.2. $\left(32^{\prime}\right)$ is also satisfied by the $\mathcal{V}_{k}$. However, while the $\mathcal{U}_{k}$ act on the commuting coordinates $z, \bar{z}$, the $\mathcal{V}_{k}$ act on the operators $W$ and $\bar{W}$ of $(\overline{6} \cdot 1 \overline{1})$. The latter, factorized by the action of the $\overline{\mathcal{V}}_{k}$ in $(6.8)$, can be pictorially identified with a sort of noncommutative coordinates on $\Sigma_{\theta}$.

Each $\gamma \neq \mathbf{1}$ in $\Gamma$ can be uniquely expressed as a positive power of a primitive element $p \in \Gamma$, primitive meaning that $p$ is not a positive power of any other $p^{\prime} \in \Gamma\left[2 \bar{z}^{2}\right]$. Let $\mathcal{V}_{p}$ be the representative of $p$. Any $\mathcal{V} \in \bar{C}^{\star}$ can be written as

$$
\mathcal{V}=\sum_{p \in\{\text { prim }\}} \sum_{n=0}^{\infty} c_{n}^{(p)} \mathcal{V}_{p}^{n}+c_{0} \mathbf{1}
$$

for certain coefficients $c_{n}^{(p)}, c_{0}$. A trace can be defined as $\operatorname{tr} \mathcal{V}=c_{0}$.
In the case of the torus one can connect the $C^{\star}$-algebras of $U(1)$ and $U(N)$. To see this one can use 't Hooft's clock and shift matrices

$$
V_{1} V_{2}=e^{2 \pi i \frac{M}{N}} V_{2} V_{1} .
$$

The $U(N) C^{\star}$-algebra is constructed in terms of the $V_{k}$ and of the unitary operators representing the $U(1) C^{\star}$-algebra. Morita equivalence is an isomorphism between the two. In higher genus, the analog of the $V_{k}$ is the $U(N)$ representation $\rho(\gamma)$ considered above. One can obtain a $U(N)$ projective unitary differential representation of $\Gamma$ by taking $\mathcal{V}_{k} \rho\left(\gamma_{k}\right)$, with $\mathcal{V}_{k}$ Abelian. This nonAbelian representation should be compared with the one obtained by the non-Abelian $\mathcal{V}_{k}$ constructed above. In this framework it should be possible to understand a possible higher-genus analog of the Morita equivalence.

The isomorphism of the $C^{\star}$-algebras is a direct consequence of an underlying equivalence between the $U(1)$ and $U(N)$ connections. The $z$-independence of the phase requires $F$ to be the identity matrix in the gauge indices. This in turn is deeply related to the uniqueness of the connection we found. The latter is related to the uniqueness of the NS connection. We conclude that Morita equivalence in higher genus is intimately related to the NS theorem.

Finally let us observe that, as our operators correspond to the $N \rightarrow \infty$ limit of projective unitary representations of $\Gamma$, these play a role in the $N \rightarrow \infty$ limit of QCD as considered in [2 $2 \overline{2}]$.

\section{Acknowledgments}

It is a pleasure to thank D. Bellisai, D. Bigatti, M. Bochicchio, U. Bruzzo, R. Casalbuoni, G. Fiore, L. Griguolo, P.M. Ho, S. Kobayashi, I. Kra, G. Landi, K. Lechner, F. Lizzi, P.A. Marchetti, B. Maskit, F. Rădulescu, D. Sorokin, W. Taylor, M. Tonin and R. Zucchini for comments and interesting discussions. G.B. is supported in part by a D.O.E. cooperative agreement DEFC02-94ER40818 and by an INFN "Bruno Rossi" Fellowship. J.M.I. is supported by an INFN fellowship. J.M.I., M.M. and P.P. are partially supported by the European Commission TMR program ERBFMRX-CT96-0045. 


\section{References}

[1] G. Bertoldi, J.M. Isidro, M. Matone and P.

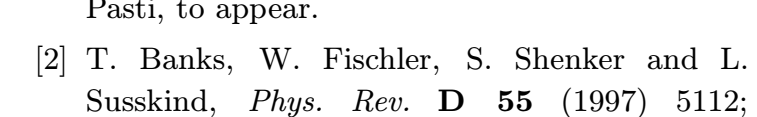

' - - - for- reviews - see- $\mathrm{A}$ - Bigatti- arrd -t:-Surskind, hep-th/9712072; T. Banks, hep-th/9911068; W. Taylor, ihep-th/9801182.

[3] N. Ishibashi, H. Kawai, Y. Kitazawa and A. - Tsüchiya, 'inuct. Phys.

[4] L. Susskind, hep-th/9704080.

[5] W. Taylor, hep-th/0002016!

[6] W. Taylor, 'Phys. Lett. B 394 (1997) 283. O. Ganor, S. Ramgoolam and W. Taylor, Nucl.' - - - - Phys. B $\mathbf{4} \overline{\mathbf{9}} \mathbf{2}(\mathbf{1} 997) \overline{1} 1 \mathrm{1}$.

[7] A. Connes, M. Douglas and A. Schwarz, $\bar{J} . \bar{H} i \bar{h}$ - - Energy Phys. 02 $(1998) 003$.

[8] M. Douglas and C. Hull, $\mathbf{0 2}(1998) 008 .$, C. Hull, 'J. High Energy Phys.'

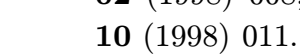

[9] D. Bigatti, IPhys. Lett. B $\mathbf{4} 51-(1999)-324$; hep-th/9802129; Ph.D. Thesis.

[10] R. Casalbuoni, 'Phys. Lett. B_ 431 (1998)_69.

[11] A. Connes, Noncommutative Geometry, Academic Press, London (1994); hep-th/0003006; G. Landi, An Introduction to Noncommutative Spaces and their Geometry, Lecture Notes in Physics: Monographs, m51, SpringerVerlag, Berlin-Heidelberg (1997); M. Douglas, hep-th/9901146.

[12] M. Douglas, H. Ooguri and S. Shenker, Phys.' Lett. B $\mathbf{4 0 2}(1997)$ 36; M. Douglas and H. Ooguri, 'Phys. Lett. B 425 (1998) 71 .

[13] N. Nekrasov and A. Schwarz, 'Comm. Math.' - Phys. 198 (1998) 689; A. Astashkevich, N. Nekrasov and A. Schwarz, ihep-th/9810147; A. Konechny and A. Schwarz, : - (1999) 561; iPhys. Lett. B $\mathbf{4 5 3}(1999) 23$.

[14] C. Hofman and E. Verlinde, J. High Energy, '- Phys. 12 (1998) 010; 'Nucl. Phys. B 547 (1999)!

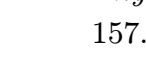

[15] B. Moriaru and B. Zumino, hep-th/9807198

[16] _. G. Landi, F. Lizzi and R. Szabo, 'Comm. Math.' ' - _ _ Phys. 206 (1999) 603.

[17] P.-M. Ho, Y.-Y. Wu and Y.-S. Wu, Phys. Rev.' ' - _ D 58 (1998)_026006; P.-M. Ho and Y.-S. Wu,

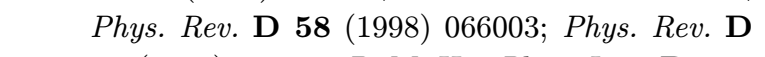
L - 60-(1999) @2G002,-P.-M- He, Pkys: Eett.-B-434 - . . (1998) 41.
[18] F. Ardalan, H. Arfaei and M.M. Sheikh-Jabbari, J. High Energy Phys. 02 (1999) 016.

[19] N. Seiberg and E. Witten, 'J. High Energy Phys.' o9 (1999)_032".

[20] V. Schomerus, 'J. High Energy Phys. $\mathbf{0} \overline{6}_{1}^{\prime}$ (1999) 030' L. Cornalba and R. Schiappa, _hep-th/9907211. L. L. Cornalba, hep-th/9909081', hep-th/9912293 Y. Imamura, IJ. High Energy_Phys. 01 (2000) 039; H. Hata and S. Moriyama, IJ. High Energy Phys. 02 (2000) 011. J. Madore, S. Schraml, P. Schupp and J. Wess, hep-th/0001203'; I.Ya. Aref'eva, D.M. Belov and A.S. Koshelev, hep-th/0001215; M.B. Green and M. Gutperle, hep-th/0002011; P. Bouwknegt and V. Mathai, hep-th/0002023: A. Matusis,_L. Susskind and N. Toumbas, hep-th/0002075: K. Hashimoto and T. Hirayama, ihep-th/0002090 A. Yu. Alekseev and A.G. Bytsko, hep-th/0002101; T. Asakawa and I. Kishimoto, hep-th/0002138; J. Ambjorn, Y.M. Makeenko, J. Nishimura, R.J. Szabo, hep-th/0002158; P.-M. Ho, hep-th/0003012.

[21] G. Bertoldi, J.M. Isidro, M. Matone and P. Pasti, to appear.

[22] G. Thompson, hep-th/930512d.

[23] F. Rădulescu, Memoirs of the American Mathematical Society 630 (1998) vol. 133; math/9912033.

[24] S. Kobayashi, Differential Geometry of Complex Vector Bundles, Iwanami Shoten Publishers and Princeton University Press, Princeton (1987); R. Gunning, Lectures on Vector Bundles over Riemann Surfaces, Princeton Univ. Notes 1967; J. Fay, Memoirs of the American Mathematical Society 464 (1992) vol. 96.

[25] M. Atiyah and R. Bott, Phil. Trans. Roy. Soc. London A 308 (1982) 524; P.G. Zograf and L.A. Takhtajan, Math. USSR Izvestiya 35 (1990) 83; D. Fine, 'Comm. Math. Phys. 140 (1991) 321; F. Ferrari, Helv. Phys. Acta 67 (1994) 702; A. Sengupta, Memoirs of the American Mathematical Society 600 (1997) vol. 126.

[26] M. Narasimhan and C. Seshadri, Ann. Math. 82 (1965) 540.

[27] S. Donaldson, Journ. Diff. Geom. 18 (1983) 269.

[28] H. McKean, Comm. Pure Applied Math. 25 (1972) 225.

[29] M. Bochicchio, 'י High Energy Phys. 01 (1999) $005:$ J. High Energy Phys. 01 (1999) 006; hep-th/990420d; inep-th/9904201: 